\title{
Subluminal and superluminal light pulse propagation under an external magnetic field in a vee-type three-level atomic medium
}

\author{
Thai Doan Thanh, ${ }^{1}$ Nguyen Tuan Anh, ${ }^{1}$ Nguyen Thi Thu Hien, ${ }^{1}$ Hoang Minh Dong, ${ }^{* 1}$ Nguyen Xuan Hao, ${ }^{2,3}$ \\ Dinh Xuan Khoa, ${ }^{3}$ Nguyen Huy Bang ${ }^{3}$ \\ ${ }^{1}$ Ho Chi Minh City University of Food Industry, Ho Chi Minh City, Vietnam, \\ ${ }^{2}$ Thu Dau Mot University, Thu Dau Mot City, Vietnam, \\ ${ }^{3}$ Vinh University, 182 Le Duan Street, Vinh City, Vietnam
}

Received December 22, 2020; accepted January 11, 2021; published March 14, 2021

\begin{abstract}
In this work, we investigate subluminal and superluminal light propagation in a vee-type three-level atomic medium under an external magnetic field. The dispersion and absorption behaviors are studied for the cases of absence and presence of a magnetic field. It is found that under an electromagnetically induced transparency condition, the light pulse can be switched between subluminal and superluminal propagation by ON-OFF switching of the magnetic field. Finally, the transient response of the medium is discussed, which shows that the considered scheme has potential applications in magneto-optic switching devices.
\end{abstract}

In recent years, the phenomenon of electromagnetically induced transparency (EIT) $[1 \div 2]$, which is generated by quantum interference between two different displacement channels and an opaque optical medium can become transparent in a probe field by applying a strong control laser field at a different frequency. The EIT effect not only reduces absorption but also enhances linear and nonlinear dispersions in the vicinity of atomic resonant frequency. One of the interesting applications of the EIT medium is that it modifies light pulse propagation through the dispersion of a medium, such as controlling and slowing down the group velocity of light, even stopping, storing, and then it retrieves light pulses $[3 \div 5]$, enhances

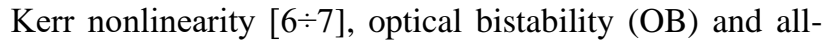
optical switching (AOS) $[8 \div 10]$, formation and optical solitons propagation $[11 \div 13]$, and so on.

In addition to intensity-controllable absorption and dispersion properties of an EIT medium, recent studies show that the optical properties of an EIT medium are also controlled by external magnetic field and polarization

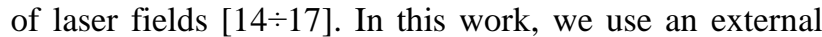
magnetic field to switch from electromagnetically induced transparency to electromagnetically induced absorption (EIA), which corresponds with the propagation of light from subluminal to superluminal velocity. We investigate the influence of an external magnetic field on the absorptive and dispersive properties as well as the group index and transient behavior of the probe field, which demonstrates that the medium can be used for optical switches at a low light intensity.

\footnotetext{
*E-mail: donghmhufi@gmail.com
}

The vee-type degenerated the atomic system under the interaction of an external magnetic field as shown in Fig.1. In this scheme, the transition $|1\rangle$ to $|3\rangle$ is applied by a weak probe laser field $\mathrm{E}_{\mathrm{p}}$ (have angular frequency $\omega_{\mathrm{p}}$ ) with the right-circularly polarized component $\sigma^{+}$. Simultaneously, a strong coupling laser field $\mathrm{E}_{\mathrm{c}}$ with the left-circularly polarized component $\sigma^{-}$(have angular frequency $\omega_{\mathrm{c}}$ ) is introduced to couple the transition $|1\rangle$ to $|2\rangle$. The medium is likely to be affected by an applied longitudinal magnetic field $B$ removing the degeneracy of the states $|2\rangle$ and $|3\rangle$, whose Zeeman shift is determined by $\Delta_{B}=\mu_{B} m_{F} g_{F} B / \mathrm{h}$, where $\mu_{\mathrm{B}}$ is the Bohr magneton, $g_{F}$ is the Lande factor, and $m_{F}= \pm 1$ is the magnetic quantum number of the corresponding state. The decay rates from the states $|3\rangle$ and $|2\rangle$ to $|1\rangle$ are given by $\gamma_{31}$ and $\gamma_{21}$, respectively. Utilizing the rotating-wave and the electric dipole approximations, the interaction Hamiltonian of the system in the interaction picture can be written as (with the assumption of $h=1$ ):

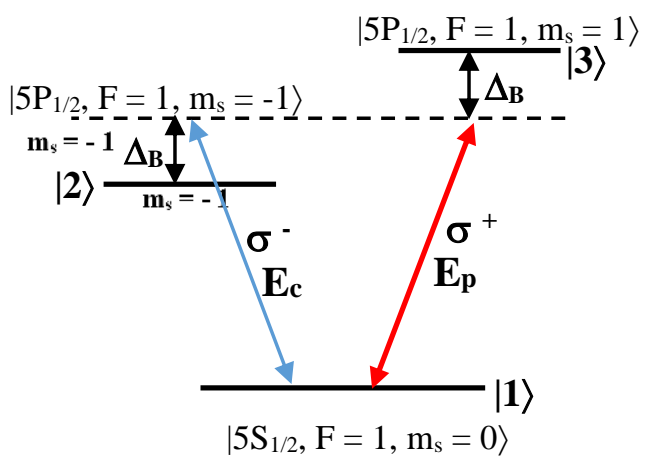

Fig.1. Schematic diagram of a vee-type degenerated atomic system under a static magnetic field and two coupling and probe laser fields.

$$
\begin{aligned}
H_{\mathrm{int}}= & -\left(\Delta_{c}+\Delta_{B}\right)|2\rangle\left\langle 2\left|+\left(\Delta_{B}-\Delta_{p}\right)\right| 3\right\rangle \\
& \langle 3|-\left(\Omega_{c}|2\rangle\left\langle 1\left|+\Omega_{p}\right| 3\right\rangle\langle 1|+H . c\right),
\end{aligned}
$$

where $\Delta_{c}=\omega_{21}-\omega_{c}$ and $\Delta_{p}=\omega_{31}-\omega_{p}$ are the frequency detunings of the coupling and probe fields and from the atomic transition frequencies, respectively. $\Delta_{\mathrm{B}}$ is the 
Zeeman shift of the levels $|2\rangle$ and $|3\rangle$ in the presence of the magnetic field (see Fig. 1) and $\Delta_{\mathrm{B}}$ is taken to zero for zero magnetic field. Under electric-dipole and rotatingwave approximations, the evolution of the system is described as follow:

$$
\begin{aligned}
& \frac{\partial \rho_{11}}{\partial t}=\gamma_{21} \rho_{22}+\gamma_{31} \rho_{33}+i \Omega_{c}^{*} \rho_{21}-i \Omega_{c} \rho_{12}+i \Omega_{p}^{*} \rho_{31}-i \Omega_{p} \rho_{13}, \\
& \frac{\partial \rho_{22}}{\partial t}=-\gamma_{21} \rho_{22}+i \Omega_{c} \rho_{12}-i \Omega_{c}^{*} \rho_{21}, \\
& \frac{\partial \rho_{33}}{\partial t}=-\gamma_{31} \rho_{33}+i \Omega_{p} \rho_{13}-i \Omega_{p}^{*} \rho_{31}, \\
& \frac{\partial \rho_{21}}{\partial t}=-\left(\frac{\gamma_{21}}{2}-i\left(\Delta_{c}+\Delta_{B}\right)\right) \rho_{21}+i \Omega_{c}\left(\rho_{11}-\rho_{22}\right)-i \Omega_{p}^{*} \rho_{23}, \\
& \frac{\partial \rho_{31}}{\partial t}=-\left(\frac{\gamma_{31}}{2}+i\left(\Delta_{B}-\Delta_{p}\right)\right) \rho_{31}+i \Omega_{p}\left(\rho_{11}-\rho_{33}\right)-i \Omega_{c} \rho_{32}, \\
& \frac{\partial \rho_{32}}{\partial t}=-\left(\frac{\gamma_{31}}{2}+\frac{\gamma_{21}}{2}+i\left(\Delta_{c}-\Delta_{p}+2 \Delta_{B}\right)\right) \rho_{32}+i \Omega_{p}^{*} \rho_{12}-i \Omega_{c} \rho_{31},
\end{aligned}
$$

where the matrix elements obey conjugated and normalized conditions, namely $\rho_{i j}=\rho_{i j}^{*} \quad(i \neq j)$, and $\rho_{11}+\rho_{22}+\rho_{33}=1$, respectively.

The relationship of the refraction index of medium $n_{\mathrm{g}}$ and the susceptibility $\chi_{31}$ is expressed as [5]:

$$
n_{g}=1+\frac{1}{2} \operatorname{Re}\left[\chi_{31}\right]+\frac{1}{2} \omega_{p} \frac{\partial \operatorname{Re}\left[\chi_{31}\right]}{\partial \omega_{p}},
$$

where $\chi_{31}=2 N\left|d_{31}\right|^{2} \rho_{31} / \mathrm{h} \varepsilon_{0} \Omega_{p}$. In accordance with the definition of the group velocity $v_{g}=c / n_{g}$ with c being the light speed in a vacuum, it can be found that the group velocity is related with the coherence term $\rho_{31}$ from Eq. (3). If $n_{g}>1$ then the group velocity is lower than the vacuum velocity, leads to subluminal propagation. Otherwise, $\mathrm{n}_{\mathrm{g}}<1$, and the field has a superluminal propagation.

With a view to illustrating applications of the model, the cold atomic medium of ${ }^{87} \mathrm{Rb}$ were applied on the $5 \mathrm{~S}$ $5 \mathrm{P}$ transitions as a realistic candidate. The designated states and the decay rates can be chosen as follows: $|1\rangle=$ $\left|5 \mathrm{~S}_{1 / 2}, \mathrm{~F}=1, \mathrm{~m}_{\mathrm{F}}=0\right\rangle,|2\rangle=\left|5 \mathrm{P}_{1 / 2}, \mathrm{~F}=1, \mathrm{~m}_{\mathrm{F}}=-1\right\rangle,|3\rangle=$ $\left|5 \mathrm{P}_{1 / 2}, \mathrm{~F}=1, \mathrm{~m}_{\mathrm{F}}=1\right\rangle$, and $\gamma_{21}=\gamma_{31}=2 \pi \times 5.3 \mathrm{MHz}$, and wavelength of the probe, coupling, $\lambda_{\mathrm{p}}=\lambda_{\mathrm{c}}=795 \mathrm{~nm}$. Landé factor $\mathrm{g}_{\mathrm{F}}=-1 / 2$ and the Bohr magneton $\mu_{\mathrm{B}}=$ $9.27401 \times 10^{-24} \mathrm{JT}^{-1},[16,18]$, respectively. It is noted that the system parameters used in this paper are scaled by $\gamma_{31}$, thus when the Zeeman shift $\Delta_{\mathrm{B}}$ is scaled by $\gamma_{31}$, then the magnetic field strength $B$ should be in units of the combined constant $\gamma_{c}=\mathrm{h} \mu_{B}^{-1} g_{F}^{-1} \gamma_{31}$.

First, the influence of the magnetic field on the absorption-dispersion behaviors of the probe field is considered by numerically solving the above density matrix equations $(2 \mathrm{a}) \div(2 \mathrm{f})$ in the steady-state and the atoms are initially assumed in the ground-state $|1\rangle$, i.e. $\rho_{11}$ $=1$. As mentioned in Fig. 2, the absorption-dispersion behaviors of the probe fields possess sensitive dependence on turning on and off of the magnetic field. Specifically, in the case when magnetic field is turn off, i.e., $B=0$ (Fig. 2a), the probe absorption can be completely suppressed, and the opaque medium becomes transparent in the probe field at the center line. The dispersion profile has a positive slope near zero detunings, which can therefore be used for sub-luminal light propagation. Contrarily, when the magnetic field B is applied (i.e., $B \neq 0$ ), the level splitting between $|2\rangle$ and $|3\rangle$ is enhanced, the quantum interference between the two quantum paths $|1\rangle \rightarrow|3\rangle$ and $|1\rangle \rightarrow|2\rangle$ is reduced, which increases the absorption of the probe field. When the external magnetic field $\mathrm{B}$ increases to a certain value $\mathrm{B}=$ $3.5 \gamma_{\mathrm{c}}$ (i.e. $\Delta_{\mathrm{B}}=3.5 \gamma_{31}$ ), the absorption of the probe field reaches a maximal value as shown in Fig. 2(b), i.e. the medium switched from EIT to EIA, the dispersion profile has a negative slope at the line center, which leads to superluminal light propagation. Such a slope of the dispersion profile can be changed from positive to negative in the presence of the magnetic field. Therefore, we can switch the group velocity of the probe field from sub- to the super-luminal domain.

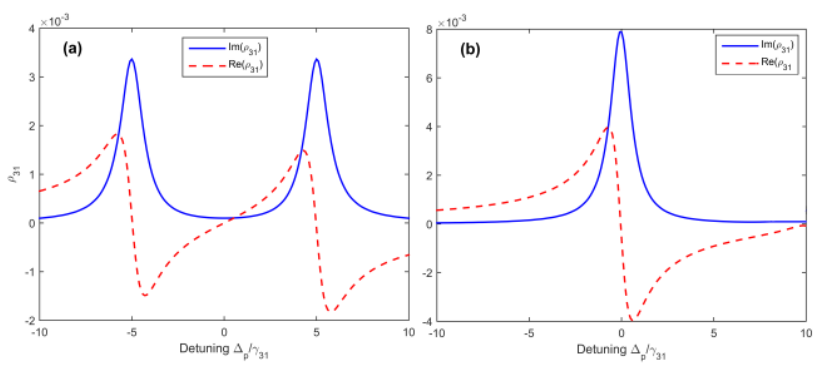

Fig. 2. Plot of the absorption-dispersion curves versus the probe detuning $\Delta \mathrm{p}$ for the absence (a) and presence (b) of the magnetic field. Other parameters are $\Omega_{\mathrm{c}}=5 \gamma_{31}, \Omega_{\mathrm{p}}=0.01 \gamma_{31}, \Delta_{\mathrm{c}}=0$, and $\gamma_{21}=\gamma_{31}$.

Next, the variation of group index versus the external magnetic field strength $\mathrm{B}$ is illustrated in Fig. 3. From Fig. 3, we showed that the group index can be controlled according to the external magnetic field. By changing the strength of the external magnetic, the magnitude and sign of the group index are controlled. Specifically, in the vicinity of the external magnetic field $\mathrm{B}=0$, the group index is positive; at the vicinity of the external magnetic field $B= \pm 3.5 \gamma_{c}$, the group index is negative, respectively. 


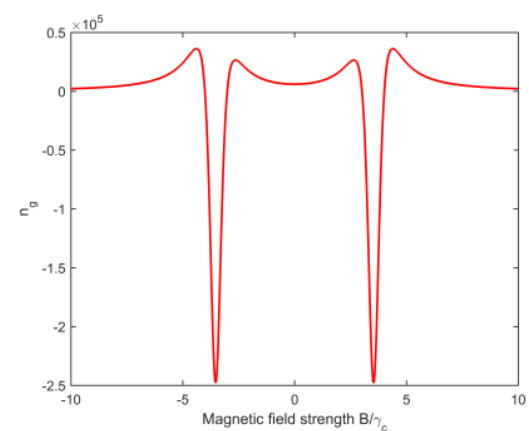

Fig. 3. Variation of group index versus the external magnetic field strength B. Other parameters are $\Omega_{\mathrm{c}}=5 \gamma_{31}, \Omega_{\mathrm{p}}=0.01 \gamma_{31}, \Delta_{\mathrm{p}}=\Delta_{\mathrm{c}}=0$, and $\gamma_{21}=\gamma_{31}$, respectively.

Now, we consider the dependence of sub- and superluminal behavior on the strengths of control and magnetic fields as shown Fig. 4. A variation of $n_{g}$ versus control Rabi frequency is presented by using Eq. (3). When the magnetic field is absent (Fig. 4a), the group index for EIT first increases to a maximum and then decreases with increasing of the control field but always corresponds to subluminal propagation. However, when the magnetic field is turned on (Fig. 4b), the large positive $n_{\mathrm{g}}$ becomes negative, which is the condition for EIA. Therefore, a valley of negative $n_{g}$ in the region of enhanced absorption is obtained. Thus an atomic medium can be tuned from sub- to super-luminal propagation by using a three-level vee-type configuration under the external magnetic field.

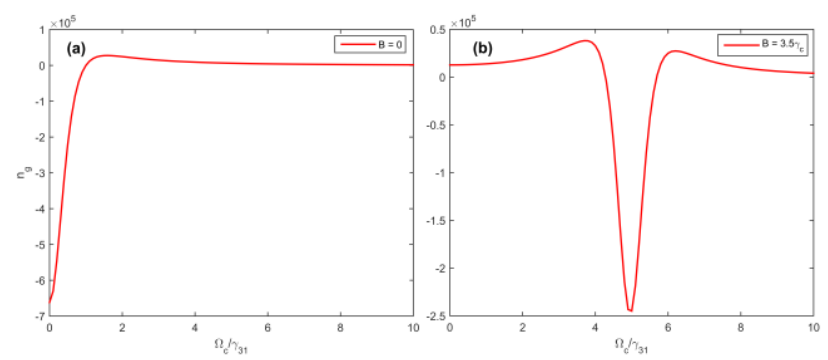

Fig. 4. The variation of group index $n_{g}$ is plotted as a function of $\Omega_{c}$, (a) $\mathrm{B}=0$ and (b) $\mathrm{B}=3.5 \gamma_{\mathrm{c}}$. Other system parameters are chosen the same as in Fig. 3.

Finally, we discuss the transient properties of the threelevel vee-type scheme. The consideration of the transient behavior of an atomic system is important due to its potential applications such as absorptive optical switching [10], in which the transmission of a highly absorptive medium is controlled by a microwave field. We show the time evolution of probe absorption $\operatorname{Im}\left(\rho_{31}\right)$ in Fig. 5. In the absence of a switching field (solid curve), the damped oscillations become steady and probe absorption reduces to zero [10]. In the case when the magnetic field is turned on the strength equal to $\mathrm{B}=3.5 \gamma_{\mathrm{c}}$, the enhanced probe absorption is observed (dashed curve). Thus, a transparent atomic medium can be switched to enhanced absorption by turning on or off the magnetic field.

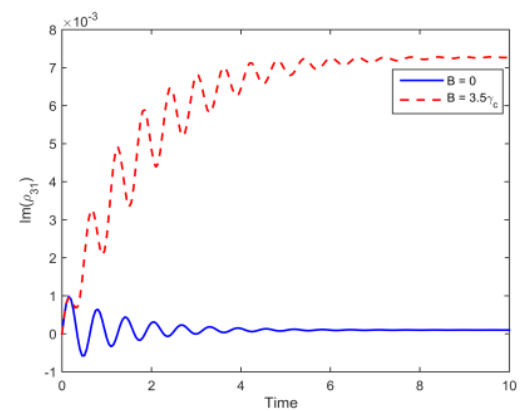

Fig. 5. Time evolution of probe absorption $\operatorname{Im}\left(\rho_{31}\right)$. Other system parameters are chosen to be the same as in Fig.2.

We have studied the dispersion and absorption properties a vee-type three-level atomic medium in an external magnetic field. It is shown that when the magnetic field is turned on, the medium can be switched from EIT to EIA. The variation of group index with control and magnetic fields showed a valley of negative group index that indicates large superluminality. Therefore, the atomic system consisting of a static magnetic field can be tuned from subluminal to superluminal propagation, which is performed by magneto-optic switches.

The financial support by Ho Chi Minh City University of Food Industry (under Contract No. 148/HD-DCT) and Vietnam Ministry of Education and Training (under grant code B2018-TDV-01SP) are acknowledged.

\section{References}

[1] K.J. Boller, A. Imamoglu, S.E. Harris, Phys. Rev. Lett. 66, 2593 (1991).

[2] M. Fleischhauer et al., Rev. Mod. Phys. 77, 633 (2005).

[3] L.V. Hau et al., Nature 397, 594 (1999).

[4] Z. Dutton, N.G.C. Slowe, L.V. Hau, Europhys. News 35, 33 (2004)

[5] V. Bharti, V. Natarajan, Opt. Commun. 392, 180 (2017).

[6] D.X. Khoa et al., J. Opt. Soc. Am. B. 31, 1330 (2014).

[7] H.R. Hamedi et al., Appl. Opt 22, 5892 (2016).

[8] A. Fountoulakis, A.F. Terzis, E. Paspalakis, Phys. Lett. A 374, 3354 (2010).

[9] D.X. Khoa et al., Opt. Soc. Am. B 33, 735 (2016).

[10] H.M. Dong, N.H. Bang, Phys. Scr. 94, 115510 (2019).

[11] H.M. Dong, L.V. Doai, V.N. Sau, D.X. Khoa, N.H. Bang, Photon. Lett. Poland 3, 73 (2016).

[12] D.X. Khoa, H.M. Dong, L.V. Doai, N.H. Bang, Optik 131, 497 (2017).

[13] H.M. Dong, L.V. Doai, N.H. Bang, Opt. Commun. 426, 553 (2018).

[14] J. Li, R. Yu, L. Si, X. Yang, J. Phys. B: At. Mol. Opt. Phys. 43, 065502 (2010).

[15] S.H. Asadpour, H.R. Soleimani, Opt. Comm. 310, 120 (2014).

[16] H.M. Dong, L.T.Y. Nga, N.H. Bang, App. Opt. 58, 4192 (2019).

[17] H.M. Dong, L.T.Y. Nga, D.X. Khoa, N.H. Bang, Sci. Rep. 1015298 (2020).

[18] D.A. Steck, Rubidium 87D Line Data, http://steck.us/alkalidata. 